\section{Visible Luminescence of Porous Silicon}

K.J. Nash, L.T. Canham and A.G. Cullis, of the Defence Research Agency, Malvern, UK, discuss the implications of recent work for theories of luminescence in porous silicon.
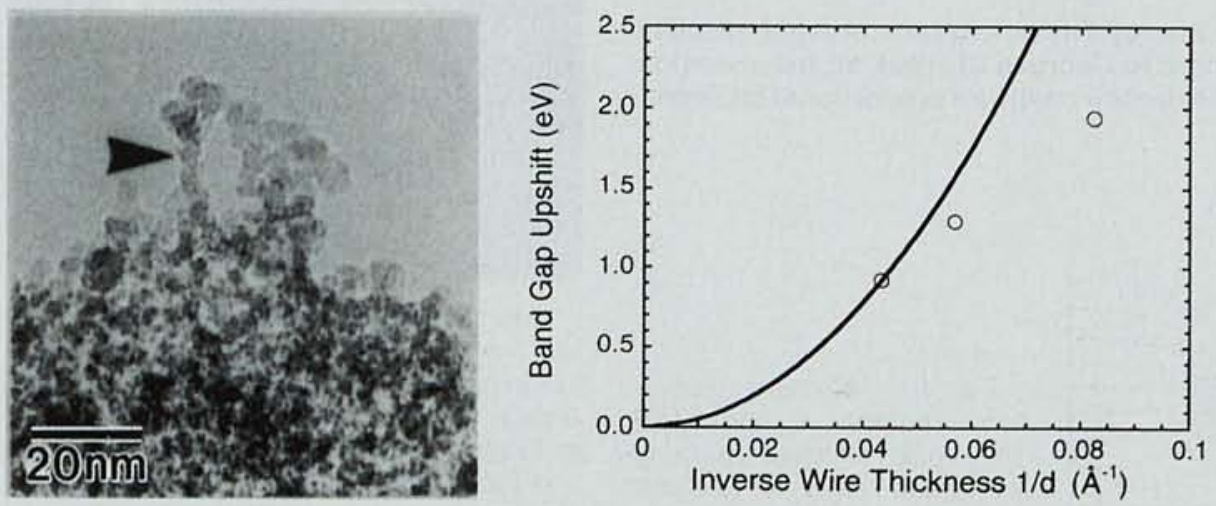

a, left) Transmission electron image of a thin layer of luminescent porous Si showing an irregular, coral-like matrix structure involving narrow silicon columns such as those (arrowed) which protrude beyond the matrix edge. The average width of the protruding columns is $\approx 30 \AA \quad[3]$. $b$, right) Upshift of the band-gap energy for [100] Si wires with (110) and (110) faces plotted versus the reciprocal of the wire thickness. The circles are first-principles calculations. The curve is the upshift calculated in effective-mass theory (EMT). The figure shows that EMT is valid for $d>23 \hat{A}\left(1 / d<0.043 \hat{A}^{-1}\right)$ but deviates from first-principles results at smaller $d$. A wire of square cross-section and side $d=27 \AA$ has the same cross-sectional area as a $30 \AA$ cylinder and, from the EMT calculation, has a band-gap upshift of $0.69 \mathrm{eV}$. This is large enough to explain the experimental PL upshift of $0.37 \mathrm{eV}$ for the wires shown in (a). The PL is upshifted less than the band-gap energy $E_{g}$ because the luminescent state is a bound exciton which has a lower energy than $E_{g}$ (From [9].)

A great deal of research has recently been devoted to understanding the efficient visible luminescence of porous silicon made by electrochemically etching a silicon wafer in hydrofluoric acid [1]. If etching is allowed to proceed until the pores coalesce, then the remaining material consists mostly of isolated $\mathrm{Si}$ columns [1] with hydride-passivated surfaces [2], and gives strong visible photoluminescence (PL). This contrasts with the behaviour of bulk crystalline $\mathrm{Si}$, whose indirect band gap of 1.11 $\mathrm{eV}$ results in very weak infra-red luminescence. Transmission electron microscopy of luminescent, re-emitting porous silicon (cover illustration) shows a fundamentally crystalline structure of undulating quantum wires with average width $\sim 30 \AA$ (see figure) and, depending upon anodisation conditions, an assembly of microcrystallites (quantum dots) in various stages of formation [3].

\section{Luminescence Mechanism}

The origin of this bright, visible PL has been hotly debated. Canham and co-workers propose that the optical properties can be explained by quantum confinement of electrons and holes in the crystalline Si skeleton, taking into account the tendency of electrons and holes to become localised by fluctuations of the wire width $[1,3]$. The crystalline Si structure can be terminated either with hydrogen or by an interface with an oxide. The hypothesis of quantum confinement is quite widely accepted, although some groups $[4,5]$ prefer to consider porous $\mathrm{Si}$ as interconnected quantum dots rather than undulating quantum wires.

Alternative theories propose that the $\mathrm{PL}$ arises, not in crystalline $\mathrm{Si}$, but in a different chemical species. The most prominent of

these alternative theories suggests [6] that the luminescence is due to the sixfold $\mathrm{Si}$ rings of $\mathrm{Si}-\mathrm{O}-\mathrm{H}$ compounds derived from the polymer siloxene $\left(\mathrm{Si}_{6} \mathrm{O}_{3} \mathrm{H}_{6}\right)$. This attribution is based upon the apparent similarity of certain properties of porous $\mathrm{Si}$ and siloxene, specifically the PL spectrum, the far-infra-red (FIR) absorption due to phonon modes, and the Raman scattering.

\section{Testing the Theories}

Recent work has strongly supported the crystalline Si model over the siloxene model for two reasons. Firstly, experimental results have been obtained that are extremely hard to reconcile with siloxene. Secondly, firstprinciples pseudopotential calculations have provided a sound theoretical basis for the crystalline-Si model.

Although the FIR absorption spectra of oxidised porous $\mathrm{Si}$ and siloxene are similar [6], the spectrum of porous $\mathrm{Si}$ is dramatically different if care is taken to avoid exposure to air $[4,7]$. The Si-O vibrational modes, which are the most prominent features in the FIR spectra of oxidised porous $\mathrm{Si}$ and siloxene, are completely absent in freshly etched porous silicon [7], which nevertheless has high radiative efficiency. As well as showing the arguments [6] in favour of the siloxene theory to be incorrect, this demonstrates that porous silicon can be luminescent even if the concentration of bonded oxygen, an essential constituent of siloxene, is extremely low [7]. It is also most unlikely that the siloxene derivative that is proposed as the source of the luminescence would survive either the hightemperature annealing [8], or the years of exposure to air [9], that luminescent porous $\mathrm{Si}$ can withstand.
First-principles pseudopotential calculations on hydrogen-passivated crystalline $\mathrm{Si}$ wires have been performed by two groups $[9,10]$. Both calculations show that quantum confinement within the Si wires increases the band-gap energy, and transforms the wires from indirect-gap to direct-gap. The values of band-gap energy (see figure) are consistent with the PL energies observed in experiments for wires whose width is known [3]. Consideration [9] of the room-temperature radiative lifetime for a $23 \AA$-thick wire shows reasonable agreement between theory ( 380 $\mu \mathrm{s}$ for a localised exciton) and experiment (65 $\mu \mathrm{s})$. The long lifetimes found both by theory and by experiment (compared to a 1 ns lifetime for a localised exciton in GaAs) indicate that the main reason for the high radiative efficiency is not the transformation from an indirect to a direct to a direct energy gap, but a remarkably low rate of non-radiative recombination. This is attributed $[1,3,9]$ to the high quality of the hydride passivation of the $\mathrm{Si}$ surface [2], and to the localisation of carriers by wire-width fluctuations, which suppresses migration to the few unpassivated non-radiative centres that remain. The crystalline-Si theory thus give a consistent explanation of the experimental energy gap, radiative lifetime, and efficiency of porous $\mathrm{Si}$. Therefore, considering the experimental observations relating to oxygen content and behaviour during high-temperature annealing, which were mentioned above, and which strongly militate against the siloxene theory, it seems extremely hard to justify the attribution of the luminescence to compounds such as siloxene.
Porous Si has inspired so much interest among semiconductor physicists that the controversy over the origin of the luminescence is unlikely to remain unresolved for very long. A great deal of new work will be presented at the forthcoming Fall Meeting of the Material Research Society in the USA. It is likely that as a consensus is reached on the physical mechanism of the luminescence, attention will increasingly shift towards technological issues, such as the design of solidstate electroluminescent devices. This would be the first step towards the ultimate goal of using porous $\mathrm{Si}$ to realise all-Si optoelectronics and high-resolution displays that are compatible with Si VLSI technology.

[1] Canham L.T., Appl. Phys. Lett. 57 (1990) 1046

[2] Yablonovitch E., et al., Phys. Rev. Lett. 57 (1986) 249.

[3] Cullis A.G. and Canham L.T., Nature 353 (1991) 335.

[4] Xie Y.H., et al., J. Appl. Phys. 71 (1992) 2403.

[5] Vial J.C. et al., Phys. Rev. B 45 (1992) 14171.

[6] Brandt M.S., et al., Solid State Commun. 81 (1992) 307.

[7] Tischler M.A. and Collins R.T., Solid State Commun. (in press).

[8] Yamada M. and Kondo K., Jpn. J. Appl. Phys. 31(1992) L993; Petrova-Koch V., et al., Appl. Phys. Lett. 61 (1992) 943.

[9] Read A.J., et al., Phys. Rev. Lett. 69 (1992) 1232.

[10] Buda F., Kohanoff J., and Parrinello M., Phys. Rev. Lett. 69 (1992) 1272. 